ein, dass Nachzügler"innen womöglich nur durch Ordnungsrecht, bspw. Mindeststandards, zu einem Politikwechsel angeregt werden können (Graf et al. 2018: 212f.). Im strengen wortwörtlichen Sinne beschreibt Up-Scaling lediglich die vertikale Dimension, in der die Länder oder der Bund die in den Kommunen erprobten Maßnahmen aufgreifen und »nach oben« tragen. Andersherum wäre ein Down-Scaling von Bundesauf Landesebene oder von Landes- auf kommunale Ebene denkbar.

Die horizontale Dimension umfasst nach Graf et al. (2018: 211ff.) Austauschformate zwischen Einheiten derselben föderalen Ebene, etwa Städtenetzwerke oder Landesarbeitsgemeinschaften. Für ein einheitliches Wording wird die Übernahme von erprobten Policies innerhalb einer föderalen Ebene als Through-Scaling bezeichnet. Da es auch dafür gewisse Ressourcen braucht - allein schon die Zeit und das Personal, um sich das Wissen über Maßnahmen an anderen Orten anzueignen -, kann es auch hier zu einer gewissen Polarisierung kommen: Einige aktive Vorreiter"innen, die voneinander lernen, stehen ressourcenschwächeren Nachzügler*innen gegenüber. Das ThroughScaling beschränkt sich also womöglich auf die Gruppe der ohnehin schon aktiven Vorreiter"innen (Graf et al. 2018: 213f.; 235).

Beim Up-, Down- und Through-Scaling scheinen Schnittstellen zum PolicyLearning, zur Diffusion und zum Transfer auf (vgl. Rose 1991; Dolowitz/Marsh 1996; Stone 2001; Dunlop 2009; Moyson/Scholten 2018). Wichtig sei an dieser Stelle dreierlei: Erstens interessieren Formen der freiwilligen Übernahme von andernorts beobachteten Maßnahmen. Zweitens muss dafür nicht unbedingt ein wechselseitiger Austausch zwischen dem Vorreiter und dem Nachzügler bestehen: Ein »Abschauen« in Gesetzestexten oder durch bestimmte Policy Entrepreneure, die im Mehrebenensystem an mehreren Stellen aktiv sind, ist auch ohne direkte Kommunikation möglich. In diesem Sinne müssen die empirischen Analysen in Kapitel 7 neben institutionalisierten Austauschformaten auch Formen des informellen Austauschs mitdenken. Und drittens geht es nicht primär darum, völlig neue Policy-Ideen zu übertragen. Interessant sind auch die inkrementellen Veränderungen innerhalb der bestehenden Policies (vgl. Instrumenten-Alternative des Typs 1 in Kapitel 4.2.2).

\title{
4.6 Akteure und ihre Handlungen
}

Bislang wurden Instrumente und Instrumenten-Alternativen, Problemstrukturen, situative Aspekte sowie Institutionen theoretisch ausgearbeitet. Das folgende UnterKapitel widmet sich nun dem in der handlungsorientierten Forschungsheuristik zentralen Erklärungsfaktor »Akteure und ihre Handlungen«. Dafür wird zuerst in das zugrundeliegende Akteursverständnis eingeführt, um daraufhin je einzelne Akteursgruppen separat aufzubereiten, denen beim barrierefreien bzw. -reduzierten Wohnraum Relevanz zugesprochen wird.

Der AEP teilt sein Akteursverständnis mit dem Akteurzentrierten Institutionalismus und denkt neben politisch-administrativen Akteuren auch außerparlamentarische Interessenvertretungen und sonstige Akteure aus Wirtschaft, Gesellschaft oder Wissenschaft mit (Böcher/Töller 2012: 191; Mayntz/Scharpf 1995a: 43). In diesem Sinne betonen Blum/Schubert (2011: 56): »Wie eng oder wie weit der Akteursbegriff gefasst werden sollte, 
muss sich letztlich immer an der Frage entscheiden: 'Wer ist tatsächlich an den politischen Prozessen beteiligt und hat Einfluss? « Weiterhin ist zwischen individuellen Akteuren - etwa: einer Ministerin oder einem Interessenvertreter - und korporativen Akteuren zu unterscheiden. Letztere schließen sich aus individuellen Akteuren mit ähnlichen Zielen zusammen und bündeln ihre Ressourcen zur Durchsetzung dieser Ziele, beispielhaft in Form einer Partei oder eines Verbands (Böcher/Töller 2012: 99; Blum/Schubert 2011: 53f.). Der Fokus dieser Arbeit liegt auf korporativen Akteuren und ihrer politischen Einflussnahme. Die Relevanz einzelner individueller Akteure wird durch das Konzept des Policy Entrepreneurs integriert, wie es prominent im MSA Verwendung findet. Damit sind Akteure gemeint, die - unter Verfolgung einer je eigenen Zielagenda - zwischen den bestehenden Interessen vermitteln (Zahariadis 2007: 74). Cairney/Zahariadis (2016: 92) betonen dabei die Langfristigkeit derartiger Strategien: »[A]dvocates lie in wait in and around government with their solutions at hand, waiting for problems to float by to which they can attach their solutions, waiting for a development in the political stream they can use to their advantage." Dies offenbart im Gegensatz zum spontanen, schnelllebigen Politikverständnis ein inkrementelles Bild politischer Prozesse und schließt damit gut an die AEP-Grundannahme zur Eigendynamik politischer Prozesse an.

Die Interessen, Ressourcen und Strategien der Akteure sieht der AEP durch institutionelle Regelungen gerahmt (Böcher/Töller 2012: 191; vgl. Kapitel 4.5). Während bestehende Institutionen tendenziell zur Beibehaltung des Status Quo beitragen, wird dem Akteurshandeln das Potential zu politischem Wandel zugesprochen (Böcher/Töller 2012: 191f.; Reiter/Töller 2014: 95). Für die Erklärung von Politikwandel erscheint es also lohnend, Akteure näher in den Blick zu fassen. Außerdem beeinflussen sich die einzelnen AEP-Erklärungsfaktoren »(über das gemeinsame Scharnier der Akteure und ihrer Handlungen) wechselseitig «(Böcher/Töller 2012: 190). Je nach ihrer Interessen- und Ressourcenlage werden Problemstrukturen gedeutet, präferierte Instrumenten-Alternativen akzentuiert oder abgelehnte in den Hintergrund gerückt. Das kennzeichnet die Eigendynamik der Akteure aus.

Um den AEP auf das eigene Forschungsdesign anzuwenden, zeigt Abbildung 6 die hier gefassten Annahmen vom einzelnen Akteur mit seinen jeweiligen Interessen und Ressourcen. Dieser tritt in Akteurskonstellationen in Interaktion mit anderen Akteuren und dies nicht nur isoliert zum Zeitpunkt X, sondern zumeist über eine einzelne Handlungssituation hinaus. Dabei nutzt er spezifische Strategien, um seine Interessen durchzusetzen.

Abbildung 6: Vom isolierten Akteur zu Akteurskonstellationen im politischen Prozess über die Zeit (orientiert an von Prittwitz 2007: 104ff.; Schneider 2003: 131ff.)

(1)

Akteure (Interessen, Ressourcen, Strategien)

\section{(2)}

Akteurskonstellation in Handlungssituationen
Politischer Prozess über die Zeit 
Interessen werden hier in Anlehnung an von Prittwitz (2007: 104) »im Sinne wohlüberlegter Zielverfolgung « verstanden (vgl. auch Sebaldt/Straßner 2004: 18; Kevenhörster 2008: 216). Ergänzt wird diese Definition um eine Unterteilung in sogenannte Veränderungsinteressen, die unzufrieden mit den bestehenden politischen Maßnahmen sind, und Status-Quo-Interessen. Dabei handeln Akteure nach Böcher/Töller (2012: 191) entweder zweckrational auf Basis ihrer Interessen - etwa: Machterhalt - oder wertrational auf Basis ihrer normativen Grundsätze. Welche Form der Rationalität überwiegt, wird durch die dem Politikfeld zugrundeliegenden Problemstrukturen beeinflusst (vgl. Kapitel 4.3). Gleichzeitig sprechen die Autor*innen Akteuren kein rein rationales Handeln zu, sondern vielmehr eine begrenzte Rationalität (Böcher/Töller 2012: 191). Simon (1972: 162ff.) erklärt dies mit der begrenzten Fähigkeit von Individuen, sich umfassend alle Informationen $\mathrm{zu}$ einem bestimmten Themenfeld $\mathrm{zu}$ erschließen. Dies wird noch erschwert durch die meist engen zeitlichen Grenzen, in denen politische Entscheider*innen sich bewegen. Dadurch werden bestimmte Instrumenten-Alternativen womöglich gar nicht identifiziert, sondern vielmehr endet der Lösungsfindungsprozess an einem ausreichend zufriedenstellenden Punkt (vgl. »satisficing«, ebenda: 168).

Entscheidungen werden also in den seltensten Fällen auf Basis vollständiger Informationen getroffen und die Aufmerksamkeit bleibt aufgrund limitierter Ressourcen notwendigerweise auf einzelne Themen beschränkt (Zahariadis 2007: 68; Wiesenthal 2018: 61f.; Sunken/Schubert 2018: 46f.; von Prittwitz 2007: 136f.). Ressourcen werden hier verstanden als »all die Mittel, Faktoren etc., die zweckmäßig zur Erreichung eines Ziels eingesetzt werden können« (Sunken/Schubert 2018: 221). Zunächst verfügt jeder individuelle Akteur über individuelle Fähigkeiten wie Charisma, Wissen oder persönliche Netzwerke. Die Ressourcen korporativer Akteure sind für die einzelnen Akteursgruppen spezifisch auszuarbeiten, wobei dem Machtressourcenansatz folgend angenommen wird, "dass die Inhalte der Politik durch die wirtschaftliche und politische Machtverteilung zwischen gesellschaftlichen Gruppen oder Klassen mit gegensätzlichen Interessen geprägt werden (Ostheim/Schmidt 2007: 40). Dabei ist von schwachen Interessen zu sprechen, wenn Akteure über weniger Ressourcen verfügen als andere (konkurrierende) Akteure, sowie umgekehrt von starken Interessen, wenn diese - relativ gesehen - über mehr Ressourcen verfügen (Gerlach 2009: 101f.; Sunken/Schubert 2018: 158).

Wichtig ist, dass die bloße Kontrolle über Ressourcen nur eine notwendige und noch keine hinreichende Bedingung darstellt (Schiffers 2016: 481f.). So ist nach Schroeder/Schreiter (2017: 374) auch die »spezifische Logik des politischen Systems « zu bedenken, entsprechend der sich eine regierende Partei Unterstützung von ihr programmatisch nahestehenden außerparlamentarischen Interessengruppen beschafft. Auch Pfadabhängigkeiten beeinflussen, inwieweit vorhandene Ressourcen effektiv zur Interessendurchsetzung genutzt werden können. Dieser Schritt ergänzt die bisherigen statischen Annahmen zu Ressourcen um eine handlungstheoretische und prozessuale Komponente der nutzbaren Strategien. Unter Strategiefähigkeit versteht von Prittwitz (2007: 117) dabei »die Fähigkeit, in Handlungssituationen im Sinne längerfristig verfolgter Ziele optimierend handeln zu können.«

Die folgenden UnterKapitel orientieren sich an dieser Struktur zu Interessen, Ressourcen und Strategien. Sie erarbeiten das Verständnis der außerparlamentarischen Interessenvertretungen (Kapitel 4.6.1), der Parteipolitik (Kapitel 4.6.2) sowie der Minis- 
terialverwaltung (Kapitel 4.6.3) und diskutieren die Rollen, die ihnen im politischen Prozess zum barrierefreien bzw. -reduzierten Wohnraum zugeschrieben werden. Da es sich mit der Interessengruppenforschung, der parteipolitischen Literatur und der Verwaltungswissenschaft um drei unterschiedliche (Teil-)Disziplinen mit unterschiedlichen Logiken handelt, variieren die Ausführungen in ihrer Struktur zum Teil leicht und setzen je spezifische Schwerpunkte.

\subsubsection{Akteure 1: Außerparlamentarische Interessenvertretungen}

Der Oberbegriff der außerparlamentarischen Interessenvertretung (auch: »Interessengruppen«bzw. lediglich »Interessen«) subsummiert in dieser Arbeit verschiedene mehr oder weniger organisierte Verbände, Unternehmen und Netzwerke. Zweckdienlich ist eine Definition in Anlehnung an van Schendelen (2014: 674) und Zimmer/Speth (2015), nach der Interessengruppen ihre Forderungen in Interaktion mit dem politischen System durchzusetzen versuchen. Das Handlungsmoment ist dabei wichtiger als die organisatorische Form. »Lobbying « wird in diesem Kontext als eine Strategie verstanden, der sich die unterschiedlichen Interessenvertretungen bedienen können. Auch wenn das Schlagwort »Lobbying« in Deutschland vielfach mit einer intransparenten, übermächtigen Einflussnahme einzelner Interessen verbunden ist (vgl. Strässer/Meerkamp 2015: 219f.; Reutter 2018: 907; Reutter 2012a: 129; Sebaldt/Straßner 2004: 15), wendet Mai (2016: 129) ein, dass Lobbying nicht nur von großen wirtschaftlichen Playern betrieben würde, sondern ebenso von Bildungsträgern, Einrichtungen der Forschung und Kultur oder Menschen mit Behinderungen. So übernehmen Interessengruppen wichtige Funktionen in demokratischen Gesellschaften, indem sie die parlamentarische parteipolitische Interessenvertretung ergänzen (Sebaldt/Straßner 2004: 15; Reutter 2012a: 129). Während sie ihr jeweiliges Spezialinteresse an das politische System herantragen - Interessenartikulation -, ist es die Aufgabe der Parteien, diese Vielzahl an Interessen zu bündeln und auszutarieren - Interessenselektion und -aggregation - (Sebaldt/Straßner 2004: 59ff.; Strässer/Meerkamp 2015: 219). ${ }^{5}$

Die folgenden Seiten führen in die außerparlamentarische Interessenvertretung im Bereich des barrierefreien bzw. -reduzierten Wohnraums ein. Nach einer Klassifikation dieser Vertretungen werden diese mit ihren jeweiligen Interessen und Ressourcen dargestellt. Daraufhin geht es um die konkret genutzten Strategien zur Umsetzung dieser Interessen.

\section{Klassifikation organisierter Interessen}

Tabelle 5 liefert einen Vorschlag, um die Bandbreite von organisierten Interessen thematisch zu strukturieren. Für den barrierefreien bzw. -reduzierten Wohnraum erfolgt im weiteren Verlauf eine Zweiteilung in die »Angebotsseite«, die Wohnraum zur Verfügung stellt, sowie die »Nachfrageseite«, die entsprechenden Wohnraum nachfragt. Erstgenannte ist am ehesten der Kategorie »Wirtschaft und Arbeit« zuzuordnen, letzt- 
genannte am ehesten der Kategorie »Soziales und Gesundheit«, dabei mit Überschneidungen zu Verbraucherschutzverbänden als "public interests«.

Tabelle 5: Klassifikation der Interessenvertretung nach inhaltlichen Kriterien (nach Sebaldt/Straßner 2004: 97ff.; Reutter 2012a: 139ff.; Rudzio 2019: 52ff.; Schroeder/Geiger 2016: 182ff.)

\begin{tabular}{|l|l|l|}
\hline Bereich & Beispiele & Eigene Ableitung \\
\hline $\begin{array}{l}\text { Wirtschaft und } \\
\text { Arbeit }\end{array}$ & $\begin{array}{l}\text { Branchen- und Berufsverbände } \\
\text { Arbeitgeber-/Arbeitnehmerverbände } \\
\text { Kammern }\end{array}$ & $\begin{array}{l}\text { Angebotsseite von } \\
\text { Wohnraum }\end{array}$ \\
\hline $\begin{array}{l}\text { Soziales und } \\
\text { Cesundheit }\end{array}$ & $\begin{array}{l}\text { Sozialleistungsverbände } \\
\text { Sozialanspruchsverbände }\end{array}$ & $\begin{array}{l}\text { Nachfrageseite nach } \\
\text { Wohnraum }\end{array}$ \\
\hline $\begin{array}{l}\text { Postmateriell \& } \\
\text { Ideell (»public } \\
\text { interests«) }\end{array}$ & $\begin{array}{l}\text { Umwelt- und Naturschutzverbände } \\
\text { Verbraucherschutzverbände }\end{array}$ & \\
\hline $\begin{array}{l}\text { Bildung, Freizeit } \\
\text { und Erholung }\end{array}$ & $\begin{array}{l}\text { Sport- und Mobilitätsverbände } \\
\text { Vereinigungen zu Weltanschauung/Religion }\end{array}$ & \\
\hline $\begin{array}{l}\text { Vertretungen } \\
\text { offentlicher } \\
\text { Cebietskörper- } \\
\text { schaften }\end{array}$ & $\begin{array}{l}\text { Deutscher Städtetag } \\
\text { Deutscher Städte- und Cemeindebund }\end{array}$ & \\
\hline
\end{tabular}

Die Angebotsseite von Wohnraum setzt sich aus Wohnungs- und Bauunternehmen, privaten Vermieter*innen sowie Architekten-, Ingenieur- und Handwerksunternehmen zusammen. ${ }^{6}$ Diese Akteure sind vielfach von wohnungs- oder sozialpolitischen Regulierungen betroffen, welche den Rahmen für wirtschaftliches Handeln entweder fördern etwa über finanzielle Anreize - oder erschweren - etwa über regulative Vorgaben oder Besteuerungen. Insofern versuchen die Verbände und Kammern als Interessenvertretungen, ihre je eigenen wirtschaftlichen Interessen in die politischen Prozesse einzuspeisen (Paster 2017: 487ff.).

Die Nachfrageseite nach Wohnraum lässt sich zunächst in Kaufinteressent"innen für selbstgenutzten Wohnraum und Mieter*innen unterscheiden. Während die erstgenannten kaum organisiert sind, werden die Interessen letztgenannter durch Mieterschutzvereine oder allgemeiner über Organisationen des Verbraucherschutzes vertreten. Daneben sind beim barrierefreien bzw. -reduzierten Wohnraum aus einer Betroffenenperspektive Verbände von Menschen mit Behinderungen sowie von älteren Menschen von Interesse. Beide Gruppen haben einen großen und langfristigen Nutzen von Wohnraum mit entsprechender Ausstattung. Andere Nutzergruppen wie Haushalte mit kleinen Kindern oder temporär körperlich Eingeschränkte - etwa nach einem Unfall oder einer Operation - sind kaum organisierbar und werden hier nicht weiter berücksichtigt.

6 Nicht zur Angebotsseite zählen Selbstnutzer*innen: diese errichten z.T. zwar in Eigenregie Wohnraum, jedoch als »Privatsache«. Vielmehr können sie als Kaufinteressent*innen der Nachfrageseite zugeordnet werden. 
Die Interessenvertretungen der Angebotsseite von Wohnraum und der Nachfrageseite nach Wohnraum verfügen je über verschiedene Ressourcen zur Durchsetzung ihrer Interessen. Neben Geld, Zeit und Wissen werden im Folgenden vier Formen von »Macht« als entsprechende Ressourcen definiert. Die Organisationsmacht beschreibt die Mitgliederstärke und damit, über welche Repräsentationsstärke eine Interessengruppe verfügt (Ebbinghaus 2019: 124). Die strukturelle Macht gibt Auskunft darüber, ob Interessengruppen gesamtgesellschaftlich relevante Güter anbieten, deren Leistungsverweigerung die politischen Akteure in Bedrängnis bringen könnten. Damit würden sie über ein glaubhaftes Drohpotential verfügen (Sunken/Schubert 2018: 159; Klenk 2019: 86). Die gesellschaftliche Macht beschreibt den Rückhalt in der Bevölkerung und letztlich fragt die institutionelle Macht nach institutionalisierten Verbindungen ins politische System (Schmalz/Dörre: 222ff.; Ostheim/Schmidt: 40).

Im Vergleich zur Angebotsseite wird die Nachfrageseite als schwaches Interesse verstanden, stellt sie doch das Gut »Wohnraum« nicht selbst bereit und verfügt schon allein dadurch über keine strukturelle Macht. Prinzipiell bescheinigt die Studienlage Nutzer*innen von Dienstleistungen oder Gütern eine schwächere Durchsetzungskraft als Leistungserbringern und -anbietern (Pappi et al. 1995; Krzywdzinski 2008; Fraune 2011; Gerlinger 2009: 33; von Winter 2014; Klenk 2018: 19f.; Wendt 2019). Jedoch müssen sich die Interessen der Angebots- und Nachfrageseite nicht zwangsweise entgegenstehen, vielmehr könnten sich spezifische wohnungspolitische Koalitionen bilden, die gemeinsame Interessen gegenüber den politischen Entscheider"innen vertreten (von Prittwitz 2007: 144). Dieses Szenario erscheint bei redistributiven Policies wie Förderprogrammen wahrscheinlich, wohingegen insbesondere regulative Vorgaben mit einem stärkeren Konfliktpotential verbunden sind (vgl. Kapitel 4.2.1).

Übergeordnete Strukturen der Interessenvertretung

Wie werden Interessen überhaupt in die politischen Prozesse transportiert? Die Interessengruppenforschung hat dazu verschiedene Paradigmen vom Pluralismus über den Korporatismus bis hin zu Netzwerk- und Lobbyismustheorien durchlaufen (vgl. Abbildung 7). ${ }^{7}$ Bei diesen Paradigmen handelt es sich um empirisch beobachtete Strukturen, die zu bestimmten Phasen dominieren. Damit ist keinesfalls verbunden, dass eine Funktionsweise die vorherige vollends abgelöst hätte. In der politischen Praxis finden sich nach wie vor Mischformen, die auch von Politikfeld zu Politikfeld variieren (Czada 2004: 47f.; von Winter/Willems 2009: 10f.; 23).

Zum weiteren Verständnis wird die pluralistische Interessenvertretung als »dynamische[r] politische[r] Wettbewerb« verstanden (Jahn 2013: 112). Hier tragen die organisierten Interessen ihre Forderungen in das politische System, welches wiederum nach den Prinzipien von Angebot und Nachfrage darauf reagiert. Kritiker*innen werfen dem Pluralismus vor, zu vernachlässigen, dass sich nicht alle Interessen gleich gut organisieren lassen. Latente Interessen - etwa: Umwelt, Demographie - sowie ökonomisch

Die Netzwerkperspektive wird hier nicht weiter verfolgt. Mit ihrem Fokus auf Strukturen ist sie für prozessorientierte Analysen nicht geeignet - so können die Interaktionen innerhalb eines Netzwerks wiederum sowohl korporatistischer als auch pluralistischer Natur sein (Sebaldt/Straßner 2004: 54f.). 
Abbildung 7: Paradigmen in der Interessengruppenforschung (eigene Darstellung)

\begin{tabular}{|l|l|l|}
\hline 1950er bis frühe 1970er & \\
\hline Pluralismus & 1970er bis 1990er \\
\hline & Korporatismus & ab 1990er \\
\hline & $\begin{array}{l}\text { Lobbyismus, } \\
\text { Netzwerktheorien }\end{array}$ \\
\hline
\end{tabular}

sekundäre Interessen - etwa: Erwerbsunfähige, Menschen mit Behinderungen - könnten sich auf diesem Wettbewerbsmarkt nicht adäquat durchsetzen (Jahn 2013: 113; Rudzio 2019: 50f.; Sebaldt/Straßner 2004: 32). Auf diese Kritik reagiert die politische Praxis mit der korporatistischen Einbindung von Interessen, wodurch schwächere Interessen gezielt gestärkt werden, etwa durch die Institutionalisierung von Beauftragten für Menschen mit Behinderungen (Rehder 2009: 269f.; Sebaldt/Straßner 2004: 41ff.; Rudzio 2019: 69f.). Zudem sollen die Ressourcen der Verbände dadurch stärker für die politische Steuerungsfähigkeit genutzt werden - in Gegenleistung für politisches Mitspracherecht (Baruth/Schnapp 2015: 247f.; Mai 2016: 121f.). Damit lösen seit den 1970er Jahren verschiedene Spielarten des Korporatismus das Paradigma des Pluralismus ab (Reutter 2012b: 17f.; Czada 2004: 37f.). Inwieweit durch diese stärkere Verflechtung von Politik und organisierten Interessen tatsächlich bestehende Machtungleichgewichte reduziert werden, ist umstritten. So unterstellt von Winter (2007: 233) der Einbindung schwacher Interessen teils einen lediglich symbolischen Charakter und laut Kevenhörster (2008: 307) pflegen korporatistische Arrangements »stets Probleme auszuklammern, die für mehrere der Beteiligten unbequem sind, und beschränken ihren Teilnehmerkreis zudem auf anerkannte Repräsentanten durchsetzungsstarker Gruppen«.

Generell erscheint die pauschale Gegenüberstellung von Pluralismus und Korporatismus ungerechtfertigt, vielmehr betonen sie unterschiedliche Aspekte - Input/Throughput; Akteure/Institutionen - und treten laut von Winter (2007: 231f.) vielfach sogar gemeinsam auf: am Rande korporatistischer Arrangements fänden sich immer wieder pluralistische Akteurskonstellationen. Im direkten Vergleich von Pluralismus und Korporatismus schlussfolgert Czada (2004: 38) deshalb: "Die Korporatismusdebatte hat Aspekte institutioneller Einbindung, strategischer Interaktion und binnenorganisatorischer Probleme in den Vordergrund gerückt, die in einflußtheoretischen Analysen zwar am Rande erwähnt, jedoch kaum theoretisch reflektiert wurden."

Seit den 1990er Jahren sind wiederum neue Dynamiken in der politischen Praxis zu verzeichnen. Der Lobbyismus wendet sich weg vom institutionalistischen und hin $\mathrm{zu}$ einem wieder verstärkt akteurs- und handlungsorientierten Blickwinkel (Reutter 2018: 907ff.). Neben der Transnationalisierung von Politik ist diese Entwicklung durch einen 
Wandel der Interessenvertretung selbst zu erklären: Einerseits bevorzugen große Unternehmen inzwischen den direkten Kontakt zu politischen Entscheider*innen anstelle des langwierigen und oft kompromissgeschüttelten Verbandswegs, andererseits findet durch spezialisierte Agenturen eine Professionalisierung statt (Reutter 2012b: 13f.; Wehrmann 2007:36f.). Die begriffliche Bestimmung von Lobbyismus und seine Abgrenzung vom Pluralismus sowie Korporatismus bleibt dabei umstritten (vgl. Töller/Böcher 2017: 538; Kleinfeld et al.: 10). Vermittelnd teilen sich Pluralismus und Lobbyismus durch ihre Handlungsorientierung einen eher informellen Charakter, wohingegen korporatistische Strukturen sich durch ihre Institutionalisierung tendenziell leichter nachvollziehen lassen. Dabei beeinflussen diese übergeordneten Strukturen der Interessenvertretung die Strategien, die von den Interessengruppen zur Durchsetzung ihrer Interessen genutzt werden.

\section{Strategien der Interessenvertretung}

Die theoretische Ausarbeitung der Strategien basiert auf der handlungsorientierten Lobbyismusforschung. Dabei ist mit Kevenhörster (2008) zwischen Inside- und Outside-Lobbying zu unterscheiden (vgl. Abbildung 8). Mit dem Erstgenannten sind direkte Beziehungen zwischen organisierten Interessen und dem politischen System gemeint, wohingegen das Letztgenannte sich außerhalb der parlamentarischen Sphäre bewegt (Kevenhörster 2008: 232).

Abbildung 8: Lobbying-Strategien (nach Kevenhörster 2008: 232; Wehrmann 2007: 50; Sebaldt/Straßner 2004: 163)

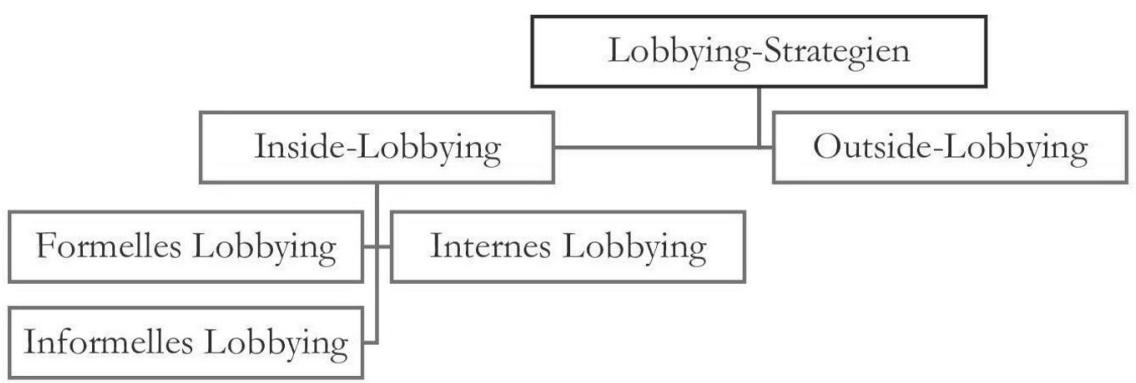

Das Inside-Lobbying wird in Anlehnung an Wehrmann (2007: 50) in formelle und informelle Einflusskanäle unterschieden. Beim formellen Lobbying können sich Verbände in einer öffentlichen Liste registrieren lassen und werden dann an Gesetzesvorhaben beteiligt (vgl. auch Sebaldt/Straßner 2004: 87f.; Reutter 2012a: 133ff.). Der zentrale Referenzpunkt ist dabei $\$ 70$ (1) der Geschäftsordnung des Deutschen Bundestages, nach dem ein Ausschuss "öffentliche Anhörungen von Sachverständigen, Interessenvertretern und anderen Auskunftspersonen « beschließen kann (Deutscher Bundestag 2019: 51). Auch die Teilnahme an Beiräten und anderen korporatistischen Arrangements ist dort festgeschrieben (Fleischer 2015). Einschränkend ist allerdings wichtig, dass die Entscheidung zur Beteiligung den jeweiligen Akteuren des politischen Systems obliegt - es besteht also kein Anhörungsrecht (Sebaldt/Straßner 2004: 144). Die einschlägige Literatur 
beurteilt diese öffentlichen Beteiligungsformate außerdem nur als begrenzt wirkmächtig, da mit dem Vorliegen eines Gesetzentwurfs bereits zentrale Vorentscheidungen getroffen seien (Sebaldt/Straßner 2004: 158ff.; Wehrmann 2007: 50f.; Strässer/Meerkamp 2015: 227ff.). Umso mehr Gewicht wird deshalb informellen Verfahren wie ministeriellen Hearings zugeschrieben. So erklärt von Beyme (2017: 234), dass Interessenvertretungen meist schon lange vor einer offiziellen Gesetzesinitiative aktiv werden und eher langfristig, inkrementell Einfluss suchen. Eine solche Einflussnahme über den schriftlichen, persönlichen oder telefonischen Austausch ist dabei keinesfalls mit Kumpanei oder gar Korruption gleichzusetzen, sondern gilt als Bestandteil des demokratischen Willensbildungsprozesses (Wehrmann 2007: 52; Strässer/Meerkamp 2015: 234). Schließlich zählt auch das interne Lobbying zum Inside-Lobbying, wobei Vertreter*innen von außerparlamentarisch organisierten Interessen Funktionen innerhalb von Parteien bekleiden (Reutter 2013: 42f.; Schroeder/Schreiter 2017: 371ff.; Rudzio 2019: 64f.).

Im Outside-Lobbying versuchen Interessenvertretungen hingegen, ihre Forderungen außerhalb vom parlamentarischen System geltend zu machen (Kevenhörster 2008: 232; Schiffers 2016: 486). Einerseits kann dies argumentativ durch die Nutzung medialer Kanäle über Stellungnahmen, Expertisen oder Pressemitteilungen geschehen, andererseits über die Formate von Protest und Skandalisierung (Töller/Böcher 2017: 538ff.; Rudzio 2019: 67f.). Insbesondere schwache Interessen, die sich auf parlamentarischem Wege wenig Einfluss versprechen, nutzen derartige Formate. Das Ziel besteht darin, öffentlichen Druck zu erzeugen und die Parteien vor dem Hintergrund ihrer Vote-Seeking-Interessen zum Handeln zu bewegen (Wehrmann 2007: 45, vgl. auch Kapitel 4.6.2).

Eng verbunden mit den Lobbying-Strategien ist die Frage, wen diese politische Einflussnahme adressiert. So liegt es in der strategischen Natur von Interessenvertreter*innen, erstens nahe an den politischen Entscheider*innen anzusetzen und zweitens in einem möglichst frühen Stadium auf Gesetzesvorhaben Einfluss zu nehmen. Insofern sind im parlamentarischen Bereich die Ausschussmitglieder zentral und in den Ministerialverwaltungen diejenigen Referate, welche die Gesetzentwürfe ausarbeiten (Reutter 2018: 912; Sebaldt/Straßner 2004: 152ff.; Wehrmann 2007: 43; von Beyme 2017: 232ff.). Aber auch einzelne oppositionelle Fachpolitiker*innen werden gezielt angesprochen, etwa, wenn Interessenvertretungen bei den Regierungsvertreter*innen kein Gehör finden oder stärkere - inhaltliche oder personelle - Bindungen zu bestimmten Parteien bestehen (Wehrmann 2007: 43; Strässer/Meerkamp 2015: 230ff.). Allzu nachvollziehbar ist schließlich die Beobachtung von Strässer/Meerkamp (2015: 231), nach der Interessenvertreter*innen dort Kontakte suchen, wo sie die größten »Schnittmengen « vermuten. Und doch darf insgesamt über der Betrachtung rein rationaler, inhaltlicher Schnittmengen das Menschliche nicht vernachlässigt werden: Sympathie - etwa über eine gemeinsame erwerbsbiographische oder regionale Herkunft - kann auch dort Beziehungen ermöglichen, wo man sie nicht vermutet. Umgekehrt verschließen Antipathien und Befindlichkeiten aus früheren Beziehungen eigentlich naheliegende Kontaktwege. 


\subsubsection{Akteure 2: Politische Parteien}

Welche Rolle kommt den parteipolitischen Akteuren beim barrierefreien bzw. -reduzierten Wohnraum zu? Parteien sind im Unterschied zu den außerparlamentarischen Interessengruppen neben der Interessenartikulation deutlich stärker auch für die Interessenaggregation, also die Bündelung unterschiedlicher Interessen, verantwortlich (Sebaldt/Straßner 2004: 59ff.; Strässer/Meerkamp 2015: 219). Sie werden hier operationalisiert über ihre Fraktionen in den Landtagen - damit zählen sowohl Oppositionsals auch Regierungsfraktionen in diese Akteursgruppe. Für den gewählten Dreiklang aus Interessen, Ressourcen und Strategien erfolgt eine Annäherung über die Lehre der Parteiendifferenz. Auf programmatischer Ebene gibt diese Aufschluss über die Interessen und vor allem über die Gründe für unterschiedliche Interessen (hier synonym zu »Positionen«) zwischen konkurrierenden Parteien. Über den Parteieneffekt als Ergebnis der Parteiendifferenz sind die den Parteien zur Verfügung stehenden Ressourcen und genutzten Strategien zu erörtern.

\section{Interessen von Parteien: Policy-, Vote- und Office-Seeking}

»Parties matter « ist die Grundaussage der Parteiendifferenzhypothese: Mit unterschiedlichen regierenden Parteien oder Parteienkoalitionen gehen unterschiedliche politische Programme, Maßnahmen - Policy Outputs - und letztlich Wirkungen Policy Outcomes - einher (Schmidt/Ostheim 2007a: 51). Diese polit-ökonomisch geprägte Literatur unterscheidet zwei Motive, warum Parteien überhaupt versuchen, sich voneinander abzugrenzen: Einerseits wollen sie die von ihnen präferierten politischen Inhalte umsetzen (Policy-Seeking), andererseits streben parteipolitische Akteure eigennützig nach Wählerstimmen zur Wiederwahl bzw. damit verbunden nach politischen Ämtern (Vote-bzw. Office-Seeking) (Wenzelburger 2015: 82; Bräuninger/Debus 2012: 28f.). In der politischen Praxis gehen indessen beide Motive vielfach ineinander über. So braucht es zur Umsetzung von programmatischen Interessen eine parlamentarische Mehrheit und dafür wiederum Wählerstimmen, umgekehrt werden nur überzeugende Wahlprogramme zur entsprechenden Mehrheit an Wählerstimmen führen (Wenzelburger 2015: 90).

Während das Policy-Seeking der Wertrationalität entspricht, weil hier die inhaltlichen oder »ideologischen« Überzeugungen handlungsleitend sind, ist das Vote- bzw. Office-Seeking der Zweckrationalität zuzuordnen (Reiter/Töller 2014: 96; Böcher/Töller 2012: 191f.). Einerseits verfolgen parteipolitische Akteure also an ihren Wertvorstellungen orientierte Interessen. Hier zeigen die Cleavage- und Milieu-Theorien, dass Parteien »von Haus aus bestimmten ideologischen Ausrichtungen zuzuordnen sind und diesbezüglich miteinander in Konflikt stehen, etwa entlang der Spannungslinien Arbeit/Kapital oder Liberalismus/Konservatismus (von Alemann et al. 2018: 140ff.; von Winter 2013: 393f.; Wehling 2006a: 99ff.; Schroeder/Geiger 2016: 184). Andererseits dürfen eigennutzgetriebene zweckrationale Interessen nicht unberücksichtigt bleiben. Die Abgrenzung gegenüber anderen Parteien - unabhängig davon, ob primär wert- oder zweckrational getrieben - wird dabei durch verschiedene Faktoren beeinflusst, wovon 
im Folgenden drei näher ausgeführt werden. Diese Faktoren sind interdependent und können sich je gegenseitig verstärken.

Der Parteienwettbewerb beschreibt »die Gesamtheit aller Handlungen politischer Parteien in demokratischen Systemen, die im Vorfeld von Wahlen mit dem Ziel des Erhalts von Wählerstimmen ausgeführt werden « (Bräuninger/Debus 2012: 9). Parteien agieren dabei nie isoliert voneinander, sondern positionieren sich in Reaktion zu ihren »Mitbewerber" innen« um Wählerstimmen. Sind die Wähler*innen stark vereinfacht auf einer LinksRechts-Achse in der Mitte konzentriert, werden die Parteien, um möglichst viele Wähler*innen für sich zu gewinnen, ihre Positionen angleichen. Sind die Wähler*innen hingegen in ihren politischen Präferenzen polarisiert und damit auf der fiktiven LinksRechts-Achse verstreut, werden Parteien je unterschiedliche Strategien entwickeln, um die je unterschiedlichen Wählergruppierungen anzusprechen - die Parteiendifferenz wäre ausgeprägter (Wenzelburger 2015: 84; Sunken/Schubert 2018: 68ff.). Die Konkurrenz um Wählerstimmen schränkt Parteien in der Entwicklung ihrer Programmatiken also ein - zumindest, sofern ihnen auch das Streben nach Mehrheiten unterstellt wird.

Mit dem Parteienwettbewerb hängt die Salienz eines politischen Problems bzw. einer konkreten Maßnahme zusammen: Wie stark wird etwas überhaupt öffentlich wahrgenommen und als Problem definiert? Zohlnhöfer (2019: 144) behauptet dazu:

»]e stärker sich Wähler für ein Politikfeld oder eine spezifische Reform interessieren, desto wahrscheinlicher wird das Politikfeld oder die Reform Einfluss auf die Wahlentscheidung haben, und desto größer wird der Anreiz für Parteien, sich den Wählerwünschen anzupassen - was wiederum zum Verschwinden von Parteiendifferenzen führt."

Dem ersten Teil dieses Gedankengangs lässt sich zweifelsohne folgen, aber ob eine erhöhte Salienz automatisch zu mehr Parteienkonvergenz führt, darf bezweifelt werden. Zohlnhöfer (2019) hat diese These für wohlfahrtsstaatliche Maßnahmen aufgestellt. Bei öffentlichkeitswirksamen und zugleich umstrittenen Themen wie etwa der Asyl- und Fluchtpolitik ist hingegen keine schwächere, sondern gerade eine stärkere Parteiendifferenz zu beobachten. Schließlich erklärt Zohlnhöfer (2019: 145f.), dass die Salienz nicht nur politikfeldabhängig variiert, sondern deutlich kleinteiliger ausfällt: In der Sozialpolitik kommt technischen Bereichen wie der Unfallversicherung oder dem Arbeitsschutz wenig Aufmerksamkeit zu. Wo Wähler*innen hingegen eine hohe eigene Betroffenheit erkennen, bspw. in der Renten- oder Gesundheitspolitik, ist eine gesteigerte Salienz anzunehmen. Vertreten Parteien dann bei einem solchen Thema dieselbe Position, spricht man von einem »valence issue« (Ruß 2014: 354).

Diffusionsprozesse sind ein dritter Faktor, der Parteiendifferenzen abschwächen kann. Setzt sich etwas parteiübergreifend als beste Lösung durch bzw. entpuppt sich umgekehrt als nicht mehr haltbare Alternative, so wird es zwangsweise zur Parteienkonvergenz kommen (Zohlnhöfer 2013: 273). Dies kann durch wissenschaftliche Erkenntnisse herbeigeführt werden, aber auch durch neue gesellschaftliche Wertvorstellungen. Allerdings ist Diffusion keinesfalls immer mit einem positiv besetzten »Lernen « gleichzusetzen: So haben manche Maßnahmen zu bestimmten Zeiten quasi Hochkonjunktur und werden parteiübergreifend geteilt - nur um einige Jahre später kollektives Policy-Versagen attestieren zu müssen. Ebenfalls unter Diffusionsprozessen, wenn auch eng mit dem Parteienwettbewerb verwoben, wird hier der von Hicks und Swank 
(1992) ausgearbeitete »Ansteckungseffekt« (»Contagion Effect«) zwischen Parteien verortet (Schmidt/Ostheim 2007a: 54f.). Der Parteienwettbewerb kann nämlich zu einer programmatischen Ausrichtung an konkurrierenden Parteipositionen führen - insbesondere, wenn die konkurrierenden Parteien eine ernstzunehmende Opposition darstellen oder als Koalitionspartner*in berücksichtigt werden müssen (Schmidt 2001: 26; Schmidt/Ostheim 2007a: 54f.; Zohlnhöfer 2019: 145).

Zusammenfassend beeinflussen verschiedene Faktoren (Parteienwettbewerb, Salienz eines politischen Problems, Diffusionsprozesse) die Entwicklung divergierender oder konvergierender Parteipositionen. Dabei muss neben dem wertrationalen, an den politischen Inhalten orientierten Handeln auch zweckrationales Handeln berücksichtigt werden.

Ressourcen und Strategien zur Durchsetzung der parteipolitischen Interessen

Inwieweit Parteien ihre Positionen letztlich in politische Maßnahmen überführen können, hängt zunächst davon ab, ob sie in Regierungsverantwortung gewählt wurden. Die Regierungsverantwortung ist deshalb so maßgeblich, weil sich prinzipiell nur mit einer Mehrheit im Parlament die eigenen Ziele und präferierten Maßnahmen umsetzen lassen. Einerseits verfügen regierende Parteien über den legitimatorisch wichtigen Vorteil einer Repräsentation des »Wählerwillens « und besitzen einen Ressourcenvorsprung durch die ihnen zugewiesenen ministeriellen Apparate (Lorenz et al. 2016: 114; von Alemann et al. 2018: 166; Saalfeld 2005: 41). Andererseits kommt ihnen die Initiativfunktion im Gesetzgebungsprozess sowie das Recht zur letzten Änderung zu, sie können Änderungsanträge blockieren und setzen die zeitliche und programmatische Agenda (Saalfeld 2005: 41; Döring 2005: 113ff.; Leunig 2016: 66). Dabei verfügen auch kleinere Regierungspartner*innen über gewichtige Machtressourcen, da sie als mitgestaltende Vetospieler*innen eine ernstzunehmende Vetomacht besitzen (Abromeit/Stoiber 2006: 70f.). Auch wenn Oppositionsparteien durchaus über Kleine und Große Anfragen Agenda-Setting betreiben (Hünermund 2018; Carstensen 2018) und Regierungsparteien gar mit ihren Inhalten »anstecken« können, so ist die Gestaltungsfähigkeit der Regierenden zweifelsohne eine größere.

Diese Gestaltungsfähigkeit wird allerdings institutionell eingeschränkt (vgl. Kapitel 4.5). So sind Konsensdemokratien wie die deutsche nicht nur mit Koalitionsbildungen und entsprechenden Koalitionszwängen konfrontiert, sondern auch Oppositionsparteien werden durch die Mitarbeit in Parlamentsausschüssen oder eine starke zweite Kammer stärkere Mitbestimmungsrechte gewährt (Tsebelis 1995; Schmidt 2001: 27ff.; Zohlnhöfer 2019: 147). Beides erschwert die Durchsetzung reiner Parteiinteressen. Damit verbunden ist auch der Wahlzyklus: Je näher eine Wahl rückt, desto geringer würden die Spielräume für grundlegende und insbesondere für unpopuläre Gesetzesvorhaben (Tufte 1980: 71ff.). Zudem beschränken auch in der Vergangenheit getroffene Entscheidungen den Gestaltungsspielraum einer Regierung. Kursabweichungen der früher eingeschlagenen »Pfade« sind immer mit Unsicherheiten über mögliche Wirkrichtungen verbunden und können bislang privilegierte Bevölkerungsgruppen verschrecken (Wenzelburger 2015: 92). Als »ideologische Pfadabhängigkeit« gelten außerdem die von einer Partei in der Vergangenheit formulierten Positionen: Zwar erlauben Lernprozes- 
se durchaus Kurskorrekturen - zu häufige oder zu einschneidende Kurswechsel beschädigen aber die Glaubwürdigkeit oder stoßen bisherige Wähler*innen vor den Kopf (Holtkamp 2012: 72, vgl. Kapitel $4.3 \mathrm{zu}$ ideologischen Filtern). Und ob Parteien ihre Programme in Regierungshandeln umsetzen können, wird schließlich auch durch das bundesstaatliche Institutionengefüge und die festgeschriebenen legislativen und exekutiven Handlungskompetenzen bestimmt (Zohlnhöfer 2019: 147; Zohlnhöfer 2013: 270; Reus/Vogel 2018: 626).

Neben diesen institutionellen Schranken, die die Durchsetzung parteipolitischer Interessen behindern können, sind verschiedene exogene Bedingungen nicht zu vernachlässigen. Dazu zählen die Wirtschaftskraft und Steuereinnahmen, aber auch die Beschäftigungsquoten und Lohnniveaus sowie demografische und milieuspezifische Strukturen (Sack/Töller 2018: 611; Leunig 2016: 67). Diese aus der sozioökonomischen Schule kommenden Annahmen beschreiben Regierungshandeln »als Reaktion auf gesellschaftliche und wirtschaftliche Entwicklungen « und schreiben Parteipolitik damit eine lediglich eingeschränkt gestaltende Rolle zu (Schmidt/Ostheim 2007b: 29; vgl. auch Obinger 2015).

\subsubsection{Akteure 3: Ministerialverwaltung}

Die Ministerialverwaltung ist ein zentraler Bestandteil des politisch-administrativen Systems. So ist jedem/jeder Minister*in eine ganze Organisation an Abteilungen und Fachreferaten zugeordnet, die sowohl an der Gesetzesvorbereitung als auch an der kontrolle maßgeblich beteiligt sind (Rudzio 2019: 250ff.; Leunig 2016: 73ff.). Die zentrale Arbeitsebene in den Ministerien sind die Referate, weshalb diesen im Folgenden die hauptsächliche Aufmerksamkeit gilt. ${ }^{8}$ Ferner bestehen deutliche Querverbindungen zum AEP-Erklärungsfaktor »Institutionen«, worin der ministerielle Zuschnitt als ein institutioneller Rahmen für das Handeln der Akteure ausgearbeitet worden ist (vgl. Kapitel 4.5.2). Diesbezüglich können die ministeriellen Akteure, die besonders relevant beim barrierefreien bzw. -reduzierten Wohnraum sind, unterteilt werden in diejenigen aus dem Sozialressort und diejenigen aus dem Bauressort.

\section{Interessen der Ministerialverwaltung}

Offiziell ist die Ministerialverwaltung die Entscheidungshelferin der Regierung, indem sie zum Beispiel Gesetzestexte vorbereitet (Berg/Cassel 2007: 306f.; Bohne 2018: 179; Fichert/Grandjot 2016: 138ff.). Nach dem Prinzipal-Agenten-Verhältnis überweist der Prinzipal - hier: die Ministeriumsspitze bzw. die Regierung - dem Agenten - hier: der Ministerialverwaltung - Aufgaben, die dieser im Sinne des Prinzipals auszuführen hat. Inwieweit orientiert sich das Verwaltungshandeln aber an den Vorgaben ihrer Prinzipale oder an anderen Beweggründen? Für dieses Rollenverständnis besteht ein Kontinuum vom unpolitischen Ausführen über ein kritisch-wissensbasiertes Beraten - kritische Loyalität, responsive Verwaltung - bis hin zum hybrid-politisierten Verwaltungshandeln (Veit et al. 2018: 414f., 433; Hustedt/Salomonsen 2014; Bogumil/Jann 2009: 186ff.;

8 Tiefergehend zur Funktionsweise und Organisation von Ministerialverwaltungen empfehlen sich Rudzio 2019: 250ff. oder Machura 2005: 35. 
Holtkamp 2012:43f.; Kropp/Ruschke 2010: 655ff.; Ebinger/Jochheim 2009: 329). Der Mittelweg der kritischen Loyalität wird auch als funktionale Politisierung bezeichnet, definiert als »die politisch vorausschauende Handlungsweise von Bürokraten« (Ebinger/Jochheim 2009: 329). Das eigenständige Agieren von Verwaltungsakteuren darf nämlich nicht mit einer prinzipiell oppositionellen Haltung zur Ministeriumsspitze gleichgesetzt werden, vielmehr kann es auch aus unspezifischen Vorgaben resultieren, aber dennoch responsiv nach dem sprichwörtlichen besten Wissen und Gewissen erfolgen (Biegelbauer 2013: 198f.; Rudzio 2019: 251).

Rückgebunden an wert- und zweckrationales Handeln entsprechen die unterschiedlichen Rollenverständnisse je einem Mischverhältnis, indem zweckrationale Interessen wie eigene Karrierebestrebungen oder Bequemlichkeit dominieren können, genauso aber der Wille zur inhaltlichen Politikgestaltung (Holtkamp 2012: 53; Bandelow/Hartmann 2015. Auch die bestmögliche Ausführung des - mutmaßlichen Willens des Prinzipals kann als ein zweckrationales Interesse konzeptualisiert werden und sich in den Rollen des unpolitischen Ausführens oder eines kritisch-wissensbasierten Beratens wiederfinden. Nach Machura (2005: 34) und Rudzio (2019: 252) überwiegt in der ministeriellen Praxis die inhaltliche Gestaltung vor Machtkampf und Eigennutzstreben (vgl. auch Böcher/Töller 2012: 191f.; Reiter/Töller 2014: 97). Diese Arbeit nimmt deshalb Abstand von der frühen ideengeschichtlichen Vorstellung einer vollends unpolitischen Verwaltung. Machura (2005: 20) folgend ist die Grenze zwischen Politik und Verwaltung ohnehin schwer zu ziehen:

»Allgemein kann gesagt werden, dass grundsätzliche Entscheidungen [...] den politischen Amtsträgern vorbehalten sein sollten [...]. Aber diese Vorstellung ist in höchstem Maße problematisch. Wer nämlich die Entscheidungsalternativen auswählt und die Informationsgrundlagen zusammenstellt, kann die politische Entscheidung durchaus lenken. Der hohe Sachverstand der kontinuierlich und spezialisiert in bestimmten Aufgabenbereichen arbeitenden Verwaltung kann hier den Ausschlag zugunsten einzelner Politiken geben.«

\section{Ressourcen der Ministerialverwaltung}

Das obige Zitat von Machura (2005) verdeutlicht die Relevanz der Ressourcen, über welche die Ministerialverwaltung - in Abgrenzung zu parteipolitischen Akteuren verfügt. Dafür wird neben der politikfeldabhängigen Bedeutung der Ministerialverwaltung das Prinzipal-Agenten-Verhältnis zwischen Politik und Verwaltung diskutiert, aber auch der ministerielle Zuschnitt und die Koordination über »Fachbruderschaften« stellen eine wichtige Ressource dar.

Nach Franz (2013: 153) lassen sich Politikfelder neben ihrem konkreten Gegenstand auch darüber charakterisieren, welches die wichtigsten Akteure in den politischen Prozessen sind. Neben parlamentarischen Politikfeldern, gekennzeichnet durch eine große politische Aufmerksamkeit, benennt er verwaltungsstaatliche und korporatistische Politikfelder. Entsprechend verfügt die Ministerialverwaltung über große Gestaltungsräume bei politischen Vorhaben mit geringer öffentlicher Aufmerksamkeit oder hoher technischer Komplexität (Veit et al. 2018: 431; Biegelbauer 2013: 213f.). Biegelbauer (2013: 57) spricht in diesem schwach politisierten Kontext von sogenannten Low-Level Set- 
tings. Eine solche Einteilung in politisch versus administrativ geprägte Politikfelder ist jedoch keinesfalls statisch. Entdecken politische Entscheider*innen durch öffentlichen Druck ein vormals administrativ dominiertes Thema für sich, sinkt die Gestaltungsmacht der Ministerialverwaltung schlagartig (Bogumil/Holtkamp 2006: 221; Biegelbauer/Grießler 2009: 61; Biegelbauer 2013: 213f.).

Am deutlichsten zeigen sich Ressourcen ministerieller Akteure aber beim PrinzipalAgenten-Verhältnis. Durch die immer komplexer werdenden politischen Probleme und die damit verbundene Spezialisierung erfährt der Agent zwangsweise einen Bedeutungszuwachs gegenüber seinem Prinzipal (Machura 2005: 41). Insgesamt wird der Ministerialverwaltung ein massiver Wissensvorsprung und damit Ressourcenvorteil gegenüber den gewählten Politiker*innen bescheinigt, noch verstärkt durch langjährige, stabile Arbeitsbeziehungen, die häufig über einzelne Legislaturperioden hinaus reichen (Heindl/Böhme 2013: 184; Schnapp/Willner 2013: 248ff.). An dieser Stelle weisen sowohl Leunig (2016: 72) als auch Wehling (2006b: 16) auf eine spannungsreiche Beziehung hin: Abgesehen von großen Politika werden Kompromisse zu Gesetzentwürfen bereits innerhalb der Ministerien ausgehandelt, womit direkt die Frage nach der Kontrolle des Agenten verbunden ist. Hybrid-politisierte Agenten könnten nämlich anders handeln, als dies von dem sie beauftragenden Prinzipal beabsichtigt ist (Schnapp/Willner 2013: 247ff.; Döhler 2005: 215ff.; Holtkamp 2012: 53f.). Die Gründe hierfür reichen - wie eingangs gezeigt - von egozentrierten zweckrationalen Interessen bis hin zur Durchsetzung politischer Inhalte, die im Widerspruch zur politischen Führung stehen (Holtkamp 2012: 53; Bandelow/Hartmann 2015). ${ }^{9}$

Ferner schreiben die institutionellen Settings der Ministerialverwaltung spezifische Ressourcen zu. Erstens definiert der ministerielle Zuschnitt im Bundesland - je nach finanzieller und personeller Ausstattung - mehr oder weniger Ressourcen für einzelne Politikfelder (Linhart/Windwehr 2012; Linhart/Raabe 2015). Und zweitens kommt der Ministerialverwaltung eine sogenannte Koordinierungsfunktion im föderalen Mehrebenensystem zu, da die Bundesländer sowohl untereinander, als auch mit der Bundesebene im Austausch stehen. Dies geschieht in formellen und informellen Gremien und vergrößert die Handlungsspielräume der Ministerialbürokratie durch entstehende Netzwerkbeziehungen (Machura 2005: 39; Schnapp 2004: 42; Manow 2005: 265f., vgl. Kapitel 4.5.3).

Allerdings unterliegt der bislang skizzierte ministerielle Ressourcenvorsprung auch einigen Einschränkungen: Ministerialbeamt*innen sind letztlich immer von Handlungsfenstern durch gewählte Politiker*innen abhängig. Nullmeier (2010: 258ff.) führt außerdem die geringe Strategiefähigkeit von Verwaltungsakteuren an: »Der Anspruch, den gerade Parteien erfüllen müssen, die Integration diverser Politikfelder mit ganz unterschiedlichen Anforderungsprofilen, übersteigt den ministeriellen strategischen Horizont « (ebenda: 262). Im AEP-Erklärungsfaktor »Institutionen«wurden dazu bereits die Möglichkeiten und Grenzen der positiven Koordination zwischen den Ressorts diskutiert

9 Zur Kontrolle der ministeriellen Apparate wird vielfach auf die politische Besetzung der Verwaltungsspitzen zurückgegriffen (vgl. Bogumil/Jann 2009: 186ff.; Vedder/Veit 2017; Heindl/Böhme 2013: 192). 
(vgl. Kapitel 4.5.1). Und schließlich unterstellen einige Autor*innen einen Bedeutungsverlust der Ministerialverwaltung durch Kürzungen sowie Auslagerungen an externe Beratungsunternehmen (Rudzio 2019: 253; Hustedt et al. 2017; Kalagi 2014; Döhler 2012). Derartige Entwicklungen beschränken die Ressourcen der Ministerialverwaltung zweifelsohne und können das Verhältnis von Prinzipal und Agent langfristig verändern.

Strategien: Bürokratisches-Agenda-Setting und strategische Interaktion

Ressourcen stellen die Basis für die Strategien dar, die ministeriellen Akteuren zur Durchsetzung ihrer jeweiligen Interessen zur Verfügung stehen. Um sich diesem Komplex konzeptionell zu nähern, unterscheiden Schnapp/Willner (2013: 250f.) das bürokratische Agenda-Setting von der strategischen Interaktion. Erstgenanntes beschreibt die Möglichkeit, dass Ministerialverwaltungen eigenmächtig Themen auf die politische Tagesordnung bringen. Strategische Interaktion wiederum heißt, dass sie im Prozess der Politikformulierung eigenmächtig gestaltend tätig werden (vgl. auch Bogumil/Jann 2009: 27; Möltgen-Sicking/Winter 2017: 21f.; Schnapp 2004: 39f.; Klenk 2019: 81f.).

Beim bürokratischen Agenda-Setting und der strategischen Interaktion ist das Verhältnis von Ministerialverwaltung und außerparlamentarischen Interessengruppen wichtig. Neben den Regierungsfraktionen stehen nämlich auch Interessengruppen im engen Austausch mit den sie betreffenden Referaten und nehmen dadurch Einfluss auf politische Prozesse (vgl. Jann/Wegrich 2003: 73). Machura (2005: 40) folgend »bestehen symbiotische Beziehungen in Bezug auf wechselseitige Informationsbeschaffung [und] gemeinsame Interessen an der Erhöhung des politischen Gewichts ihres Bereiches« (vgl. Baruth/Schnapp 2015: 245ff.; Mai 2016: 121ff.). Diese sogenannten Fachbruderschaften führen dazu, dass Gesetzentwürfe bereits frühzeitig - und häufig unterhalb des politischen Radars »verbandsfest« gemacht werden (Rudzio 2019: 66; Machura 2005: 40).

\subsection{Zusammenfassung des Analyserahmens}

Kapitel 4 hat in den Ansatz eigendynamischer politischer Prozesse als Forschungsheuristik eingeführt und die fünf Erklärungsfaktoren »Instrumente und InstrumentenAlternativen «, »Problemstrukturen«, »situative Aspekte«, »Institutionen« sowie »Akteure und ihre Handlungen« theoretisch ausgearbeitet. Die Interdependenzen zwischen diesen Faktoren sowie die zufälligen Entwicklungen innerhalb einzelner Faktoren werden über das Konzept der Eigendynamik erfasst. Letztlich erzeugt dieses Wechselspiel Rückwirkungen auf die tatsächlich durchgesetzten Policies und damit auf das, was für den Untersuchungsgegenstand des barrierefreien bzw. -reduzierten Wohnraums erklärt werden soll.

Abbildung 9 veranschaulicht die dafür vorgenommenen theoretischen Schwerpunktsetzungen. So wurden die Instrumente und Instrumenten-Alternativen in regulative, ökonomische, kooperative und informationelle Instrumente unterteilt und damit definiert, über welche Steuerungsmöglichen politische Entscheider*innen überhaupt verfügen. Mithilfe dieser Typologie lässt sich nicht nur der bestehende Policy-Mix zum barrierefreien bzw. -reduzierten Wohnraum operationalisieren, sondern auch 